\section{Autofocused Spotlight SAR Image Reconstruction of Off-Grid Sparse Scenes}

\author{
SEDAT CAMLICA \\ TOBB University of Economics and Technology, and Aselsan A.S., \\ Ankara, Turkey
}

ALI CAFER GURBUZ, Member, IEEE

Utah State University, Logan, USA

ORHAN ARIKAN, Member, IEEE

Bilkent University, Ankara, Turkey

\begin{abstract}
Synthetic aperture radar (SAR) has significant role in remote sensing. Phase errors due to uncompensated platform motion, measurement model mismatch, and measurement noise can cause degradations in SAR image reconstruction. For efficient processing of the measurements, image plane is discretized and autofocusing algorithms on this discrete grid are employed. However, in addition to the platform motion errors, the reflectors, which are not exactly on the reconstruction grid, also degrade the image quality. This is called the off-grid target problem. In this paper, a sparsity-based technique is developed for autofocused spotlight SAR image reconstruction that can correct phase errors due to uncompensated platform motion and provide robust images in the presence of off-grid targets. The proposed orthogonal matching pursuit-based reconstruction technique uses gradient descent parameter updates with built in autofocus. The technique can reconstruct high-quality images by using sub Nyquist rate of sampling on the reflected signals at the receiver. The results obtained using both simulated and real SAR system data show that the proposed technique provides higher quality reconstructions over alternative techniques in terms of commonly used performance metrics.
\end{abstract}

Manuscript received March 31, 2016; revised November 1, 2016; released for publication February 15, 2017. Date of publication February 24, 2017; date of current version August 7, 2017.

DOI. No. 10.1109/TAES.2017.2675138

Refereeing of this contribution was handled by R. M. Narayanan.

This work was supported by TUBITAK within 1001 research program Grant project 113E515.

Authors' addresses: S. Camlica is with the Department of Electrical and Electronics Engineering, TOBB University of Economics and Technology, Ankara, 06560, Turkey, and also with Aselsan A.S., Ankara, 06370, Turkey, E-mail: (scamlica@aselsan.com.tr); A. C. Gurbuz is with the Department of Electrical and Computer Engineering, Utah State University, Logan, UT 84322, USA, E-mail: (alicafer@ieee.org); O. Arikan is with the Department of Electrical and Electronics Engineering, Bilkent University, Ankara 06800, Turkey, E-mail: (oarikan@ee.bilkent.edu.tr).

0018-9251 (C) 2017 IEEE

\section{INTRODUCTION}

Synthetic aperture radar (SAR) [1] provides highresolution images of ground reflectivity by processing multiple returns acquired on a flightpath of its platform effectively synthesizing a very large aperture antenna. Because of this high resolution capability in both range and crossrange directions and robustness to adverse weather conditions, SAR has great importance and wide usage areas ranging from civilian applications, such as environmental monitoring, terrain mapping, to military areas, such as surveillance and intelligence missions. In SAR systems, range resolution is determined by the bandwidth of the system and high resolution requirements mean directly an increase in bandwidth and correspondingly increases in analog to digital converter (ADC) rates, total number of measurements, required memory, and processing power. In classical SAR systems, the SAR signal uses a contiguous bandwidth and the cross range is uniformly sampled. However, in the case of increase in the bandwidth, it is possible that some frequency subbands may be used by other systems such as communication or it might be the case where no transmission is allowed. In some cases, the data obtained at random cross range points may be corrupted or may not be obtained if the same antenna is used for another task for a multifunction radar system. These types of acquisition modalities may necessitate nonuniform sampling in cross range as well. All of these different reasons and developments require SAR systems to be able to generate high-quality and high-resolution images from possibly low number of measurements that may be subsampled both in range and cross-range direction.

Compressive sensing (CS) [2], [3] techniques provide valuable solution alternatives to the problems of SAR systems. Unlike classical SAR imaging techniques, CS methods can operate on sub Nyquist rate data to reconstruct SAR images if imaging scene is sparse in a certain known transform domain. Due to very appealing properties of CS and its possibly important advantages for radar, CS-based techniques have received considerable attention both in the radar and SAR research communities for the last few years. Extensive literature and further details on CS-based radar and SAR applications can be found in the excellent review papers on the topic [4], [5].

CS theory provides strong results, which guarantee stable solution of the reconstructed sparse target scenes under a perfectly known sparsity basis satisfying certain additional constraints [6]. In order to form the synthetic aperture, precise relative position (such as distance, bearing, etc.) information between the SAR sensor platform, and the area of the interest are necessary. On the other hand, because of the inaccuracy in the navigation systems, uncompensated motion errors always exist, resulting in phase errors in the received SAR signal. In addition to the platform motion errors, system modeling errors such as discretization of the SAR imaging problem and assuming reflectors on the centers of the discrete image patches while they can be anywhere also create mainly phase mismatches. Total residual 
phase errors are the main cause of the degradations in highresolution SAR images, limiting achievable performance especially in the cross-range direction.

Methods for correcting phase-dependent errors due to uncompensated platform motion errors in SAR imaging are called autofocus techniques. There are various types of approaches to autofocus problem [7]-[11]. Classical autofocus approaches such as phase gradient autofocus (PGA) [7] use the data acquired at Nyquist-rate or above and phase error estimation is generally done as a post processing step on the reconstructed defocused images reconstructed by conventional techniques, such as polar-format algorithm (PFA) [1], [12]. It is observed that the performance of these techniques degrades under sub Nyquist rate data. On the other hand, CS-based formulation of the SAR imaging problem allows more generalized subsampling data acquisition schemes while providing prior information such as sparsity through regularization and a general joint imaging and autofocus framework. CS-based SAR imaging with autofocus has been studied in the literature by several papers [9]-[11], [13]-[16].

Sparsity-based SAR imaging and autofocus techniques generally incorporate the system model and the data into different types of optimization problems. Hence in these techniques, autofocus is handled during the process of image formation rather than in post processing as done in classical techniques, such as PGA. A sparsity driven autofocus (SDA) technique is used in [9], where an optimization problem in which the cost function is composed of a data fidelity term and a regularization term which is the $\ell_{1}$ norm of the discretized imaging field. The optimization problem is solved with respect to the image and the phase error through iterative separate minimizations over each parameter. Similarly in [10], an optimization problem with some practical benefits compared to [9] is proposed, where the data fidelity term is minimized with an additional constraint on the $\ell_{1}$ norm of the discretized imaging field is less than a given threshold. An expectation maximization matching pursuit (EMMP)-based iterative SAR imaging and autofocus technique is proposed in [11], where again the data fidelity term is minimized with the constraint of the sparsity level or the $\ell_{0}$ norm of the image being less than a predefined threshold, while in [9] and [10], the phase estimate is obtained following the reconstruction of the entire image in each iteration, in EMMP the image and the phase estimates are obtained iteratively through a matching pursuit procedure. Other techniques on the issue [13]-[16] follow the same concept with minor variations.

All of the discussed techniques actually require scenes that admit exact sparse representation in some known dictionaries and they deal with only the platform motion-based phase errors. However, another source of error that invalidates the exact sparsity assumptions and affect the reconstruction performance of sparsity-based techniques is the basis mismatch. These techniques discretize the image space and assume that the scene is sparse on the discretized grid. However, the scattering centers can reside anywhere in the continuous image domain rather than the assumed discrete grid locations causing basis mismatch and degradation in SAR images: a problem which is not considered by the alternative techniques mentioned above. This issue is known as the off-grid target problem in sparse representation and CS literature and is faced not only in SAR imaging but also in frequency estimation [17], angle of arrival estimation [17], and delay-doppler imaging [18]. There are various types of solutions to off-grid target problem in CS [18][22]. Among these techniques, the parameter perturbationbased orthogonal matching pursuit (PPOMP) proposed in [18], reconstructs signals where the sparsity of the signal is in a continuous parameter space and the sparsity basis is constructed through discretization of this parameter space. PPOMP technique jointly solves for the parameter perturbations from the grid centers and the amplitudes for the atoms iteratively using a gradient descent type parameter search.

The proposed algorithm named as perturbed autofocus SAR (PA-SAR), is developed for the spotlight SAR imaging mode and generates sparse SAR images by jointly solving for autofocus and off-grid target errors. The current autofocus techniques do not take care of off-grid target errors, and the proposed PA-SAR technique, as to best of our knowledge, is the first technique that does SAR imaging under both autofocus and off-grid target errors jointly. PA-SAR uses an Orthogonal matching pursuit (OMP) framework in image generation steps, and applies parameter perturbation as PPOMP to have better estimates of off-grid scatterer locations and jointly estimates the phase errors due to platform motions with the perturbed target locations. Preliminary results are given in [23]. The technique allows reduced number of measurements and results in sparse SAR images. The results obtained using both simulated and real SAR system data show that the proposed technique provides better SAR image reconstructions in terms of commonly used performance metrics.

The organization of this paper is as follows. The spotlight SAR signal and phase error models are introduced in Section II. The detailed derivation of the proposed technique is given in Section III. Results are provided in Section IV and conclusions are drawn in Section V.

\section{SPOTLIGHT MODE SAR SIGNAL MODEL}

In spotlight mode SAR, typically a linear frequency modulated (FM) chirp signal $s(t)$ is transmitted with a certain pulse repetition interval $\left(T_{\mathrm{PRI}}\right)$

$$
s(t)=e^{j\left(\omega_{0} t+a t^{2}\right)}, \quad|t| \leq 0.5 T
$$

where $\omega_{0}$ is the RF carrier frequency in radians, $2 a$ is the FM rate, and $T$ is the pulse duration, which is a small fraction of $T_{\mathrm{PRI}}$. For each pulse $i$, the received signal from the terrain of interest $y_{i}(t)$ is given by [24]

$$
\begin{aligned}
& y_{i}(t) \\
& =\iint_{\sqrt{z_{1}^{2}+z_{2}^{2}} \leq Z_{L}} x\left(z_{1}, z_{2}\right) e^{-j \Omega(t)\left(z_{1} \cos \left(\varphi_{i}\right)+z_{2} \sin \left(\varphi_{i}\right)\right)} d z_{1} d z_{2}
\end{aligned}
$$


where $x\left(z_{1}, z_{2}\right), \Omega(t), \varphi_{i}$, and $Z_{L}$ are the ground reflectivity, the radial spatial frequency, corresponding look angle of the $i$ th pulse, and length of the ground patch, respectively.

High resolution SAR image reconstruction for arbitrary terrain reflectivity distributions requires Nyquist rate or above sampling of the return signals and coherent processing of all the available samples. However, especially in applications where the goal is to image a few man-made targets of interest with strong reflectivity with respect to the reflectivity of the ground patch, CS techniques offer an alternative image reconstruction approach where below Nyquist rate sampling suffices for high-resolution image reconstructions. To develop the underlying idea, we use the following reflectivity model for the terrain patch of interest:

$$
x\left(z_{1}, z_{2}\right)=\sum_{k=1}^{K} \alpha_{k} \psi\left(z_{1}-z_{1 k}, z_{2}-z_{2 k}\right)
$$

where $\mathrm{K}$ is the number of dominant scattering centers, $\alpha_{k}$ 's are their complex valued reflectivities, $\left(z_{1 k}, z_{2 k}\right)$ 's their respective positions in the scene, and $\psi\left(z_{1}, z_{2}\right)$ is a highly localized two-dimensional (2-D) function that is chosen to represent complex man-made targets by superposition of localized scattering centers. As long as the spatial-support of the $\psi\left(z_{1}, z_{2}\right)$ is limited to a resolution-cell dimension, the SAR reconstructions are known to be insensitive to the actual shape of the $\psi\left(z_{1}, z_{2}\right)$ [24]. Under this model, received signal can be rewritten as

$$
\begin{aligned}
y_{i}(t)= & \iint\left(\sum_{k=1}^{K} \alpha_{k} \psi\left(z_{1}-z_{1 k}, z_{2}-z_{2 k}\right)\right) \\
& \times e^{-j \Omega(t)\left(z_{1} \cos \left(\varphi_{i}\right)+z_{2} \sin \left(\varphi_{i}\right)\right)} d z_{1} d z_{2}
\end{aligned}
$$

or much simply as

$$
y_{i}(t)=\sum_{k=1}^{K} \alpha_{k} g_{i}\left(t ; z_{1 k}, z_{2 k}\right)
$$

where $g_{i}\left(t ; z_{1 k}, z_{2 k}\right)$ is the equivalent SAR operator function. In this form of the received signal, total of $3 \mathrm{~K}$ unknown parameters of scene reflectivity are in multiplicative form: $\alpha_{k} \psi\left(z_{1}-z_{1 k}, z_{2}-z_{2 k}\right)$ preventing us to have a linear relationship between measurements and the unknown parameters. One commonly used technique employs a uniform grid on the terrain patch and restricts the scattering centers to reside on the grid positions: $\left(\bar{z}_{1 n}, \bar{z}_{2 m}\right)$, where $\bar{z}_{1 n}=\left(n-\frac{N}{2}\right) \Delta z_{1}$, and $\bar{z}_{2 m}=\left(m-\frac{M}{2}\right) \Delta z_{2}$, for $1 \leqslant n \leqslant$ $N$ and $1 \leqslant m \leqslant M$, respectively. Then the received signal can be modeled as

$$
\begin{aligned}
y_{i}(t)= & \iint \sum_{n=1}^{N} \sum_{m=1}^{M} \overline{\mathbf{x}}(n, m) \psi\left(z_{1}-\bar{z}_{1 n}, z_{2}-\bar{z}_{2 m}\right) \\
& \times e^{-j \Omega(t)\left(z_{1} \cos \left(\varphi_{i}\right)+z_{2} \sin \left(\varphi_{i}\right)\right)} d z_{1} d z_{2}
\end{aligned}
$$

where $\overline{\mathbf{x}}(n, m)$ is 2-D ground reflectivity matrix of size $N \times M$ and assumes nonzero values only on those unknown grid positions, where the $K$ scattering centers reside. Therefore, in this form the $K$-sparse unknowns are embedded in an $N \times M$ dimensional space. This lifting to higher dimensional space enables us to represent the relationship between measurements and unknowns in a linear form. The assumptions of scattering centers residing on the grid locations can cause significant degradation in actual scene reconstructions. This phenomenon is known as the "off-grid problem" which is not an exception but the rule in SAR reconstruction.

One brute force approach to gain resiliency against the off-grid problem is to use finer grid sizes which implies larger grid dimensions and embedding into very highdimensional spaces that require close to Nyquist rate sampling for reliable reconstructions. That limit is the classical SAR reconstruction which is well known and well documented. Therefore, it is important to tackle the off-grid problem over reasonable sized grids.

Let $\mathbf{C}_{i}^{\prime}$ be a matrix with entries; $\mathbf{C}_{i}^{\prime}\left(n^{\prime}, m^{\prime}\right)=$ $\exp \left(-j \Omega\left[n^{\prime} \Delta t\right]\left(z_{1 m^{\prime}} \cos \left(\theta_{i}\right)+z_{2 m^{\prime}} \sin \left(\theta_{i}\right)\right)\right), \quad$ where $\Omega\left[n^{\prime} \Delta t\right]$ is the time sampled radial spatial frequency, $\quad \sqrt{z_{1 m^{\prime}}+z_{2 m^{\prime}}}<Z_{L}, \quad 0 \leq n^{\prime} \leq T \cdot f_{s}-1 \quad$ and $1 \leq m^{\prime} \leq N M, f_{s}$ is the sampling rate in Hz. Then, the discretized version of the observation kernel in (6), $\mathbf{C}_{i}$ is formed by taking column-wise inverse discrete Fourier transform (DFT) of $\mathbf{C}_{i}^{\prime}$ [1], [14], [25]. The complex valued discrete SAR projection operator $\mathbf{G}_{i}$ at cross range $i$ is given by $\mathbf{G}_{i}=\mathbf{F}^{-1} \mathbf{C}_{i}$, where $\mathbf{F}$ is a convenient DFT matrix.

Using $\mathbf{G}_{i}$ and rewriting the expressions in vector form yields a linear formulation of received signal as

$$
\mathbf{y}_{i}=\mathbf{G}_{i} \mathbf{x}
$$

where $\mathbf{x}$ is the column stacked version of the $\overline{\mathbf{x}}$ in (6) and referred as the discrete ground reflectivity vector of size $N M \times 1, \mathbf{y}_{i}$ is the sampled return signal at cross range $i$ by sampling fast time $t$ to produce $L$ measurements. At this point, it should be noted that the received signal can be sampled by a classical ADC or a compressive receiver as in [26]. Nevertheless, this is modeled in $\mathbf{G}_{i}$, which is the complex valued discrete SAR projection operator for the cross range position $i$ relating the received measurements $\mathbf{y}_{i}$ to the unknown ground reflectivity vector.

SAR data processing need to know the distance and the angle between the SAR platforms and the scene center. However, due to reasons such as limited accuracy of navigational sensors, there is always a certain level of uncompensated platform motion error, which cause phase uncertainties in the received signal. If the uncertain position of the platform is assumed constant over a single pulse duration at an aperture position but vary in cross-range direction, then a phase error which is constant for fast time but vary only in cross-range can be assumed. Under such an assumption and in the presence of measurement noise, $\mathbf{y}_{i}$ can be written as

$$
\begin{aligned}
\mathbf{y}_{i} & =\operatorname{diag}\left[e^{j \phi_{i}}, e^{j \phi_{i}}, \ldots, e^{j \phi_{i}}\right] \mathbf{G}_{i} \mathbf{x}+\mathbf{w}_{i} \\
& =\Phi_{i} \mathbf{G}_{i} \mathbf{x}+\mathbf{w}_{i}
\end{aligned}
$$


where $\Phi_{i}$ is an unknown $L \times L$ diagonal matrix with only diagonal entries being the phase error $e^{j \phi_{i}}$ and $\mathbf{w}_{i}$ is the additive complex white Gaussian measurement noise vector.

If we have D cross-range samples, the combined measurements can be written as

$$
\left[\begin{array}{c}
\mathbf{y}_{1} \\
\mathbf{y}_{2} \\
\vdots \\
\mathbf{y}_{D}
\end{array}\right]=\left[\begin{array}{llll}
\Phi_{1} & & & \\
& \Phi_{2} & & \\
& & \ddots & \\
& & & \Phi_{D}
\end{array}\right]\left[\begin{array}{c}
\mathbf{G}_{1} \\
\mathbf{G}_{2} \\
\vdots \\
\\
\end{array}\right.
$$

or simply as $\mathbf{y}=\Phi \mathbf{G x}+\mathbf{w}$, where $\mathbf{y}$ is an $L D \times 1$ vector of combined measurements, $\Phi$ is $L D \times L D$ diagonal matrix having the unknown phase error terms, $\mathbf{G}$ is the equivalent $L D \times N M$ discrete SAR operator, and $\mathbf{w}$ is the combined noise vector which is white Gaussian noise (WGN) assuming independent measurement noise for different aperture locations.

\section{PROPOSED METHOD}

For a $K$ sparse scene of reflectivity modeled as in (3), the sampled combined measurements can be expressed as

$$
\mathbf{y}=\Phi \sum_{k=1}^{K} \alpha_{k} \mathbf{g}\left(f_{1, k}^{o}, f_{2, k}^{o}\right)+\mathbf{w}
$$

where $\alpha_{k}$ is the complex reflectivity of the $k$ th target, and $\mathbf{g}\left(f_{1, k}^{o}, f_{2, k}^{o}\right)$ is the combined SAR operator for all cross range positions for a target at position $\boldsymbol{\theta}_{k}^{o}=\left(f_{1, k}^{o}, f_{2, k}^{o}\right)$. If the target were exactly on the grid, then $\mathbf{g}($.$) would be the$ column of $\mathbf{G}$ matrix corresponding to that grid location. However, a target with parameters $\boldsymbol{\theta}_{k}^{o}$ may not be located exactly at a grid but is positioned close to a grid node with an unknown perturbation as $f_{1, k}^{o}=f_{1, k}+\delta f_{1, k}$ and $f_{2, k}^{o}=$ $f_{2, k}+\delta f_{2, k}$, where $\boldsymbol{\theta}_{k}=\left(f_{1, k}, f_{2, k}\right)$ are the nearest grid position, with perturbations $\left|\delta f_{1, k}\right|<0.5 \Delta_{1}$ and $\left|\delta f_{2, k}\right|<$ $0.5 \Delta_{2}$ with $\Delta_{1}$ and $\Delta_{2}$ represents the grid dimensions in range and cross-range, respectively.

High-quality image reconstruction requires estimation of both the unknown phase errors in $\Phi$ and image parameters $\alpha_{k}$ and $\left(f_{1, k}^{o}, f_{2, k}^{o}\right)$ including the grid perturbations. Hence, the goal is to apply autofocus and handle the offgrid problem simultaneously. To achieve this goal, the following optimization problem is formulated

$$
\begin{aligned}
& \min _{\alpha_{k}, \delta f_{1, k}, \delta f_{2, k}, \phi_{i}}\left\|\mathbf{y}-\Phi \sum_{k=1}^{K} \alpha_{k} \mathbf{g}\left(f_{1, k}+\delta f_{1, k}, f_{2, k}+\delta f_{2, k}\right)\right\|_{2} \\
& \text { s.t. } \phi_{i}=\angle\left(\sum_{k=1}^{K} \alpha_{i, k} \mathbf{g}_{i}\left(f_{1, k}+\delta f_{1, k}, f_{2, k}+\delta f_{2, k}\right)\right)^{H} \mathbf{y}_{i} \\
& i=1,2, \ldots D \text { and }\left|\delta f_{1, k}\right|<\Delta_{1} / 2,\left|\delta f_{2, k}\right|<\Delta_{2} / 2
\end{aligned}
$$

where $i$ denotes the pulse number. The goal in (11) is to minimize the $\ell_{2}$ norm of the residual error of the measurements with constraints being the phase error equal to the minimizer of the per pulse cost as defined in [14] and the perturbations being within a grid cell. The optimization problem in (11) is nonconvex and poses a significant
TABLE I

Proposed Algorithm

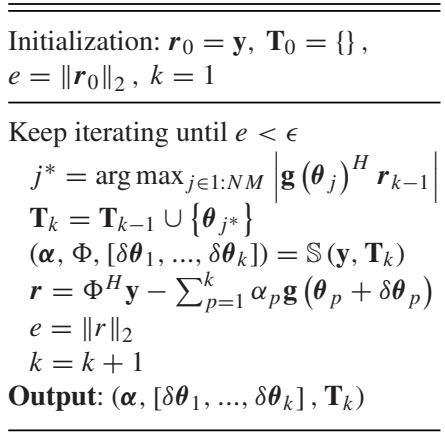

challenge to solve directly. Instead, a suboptimal iterative method which alternatively solves for the phase errors and the target perturbations within a greedy technique is developed. The outer shell of the proposed method is based on OMP [27] and given in Table-I.

At the $k$ th iteration of the proposed algorithm, the selected $k$ grid locations $\mathbf{T}_{k}$ and the measurements are input to an inner algorithm abstractly shown as

$$
\left(\boldsymbol{\alpha},\left[\delta \boldsymbol{\theta}_{1} \ldots \delta \boldsymbol{\theta}_{k}\right], \Phi\right)=\mathbb{S}\left(\mathbf{y},\left[\begin{array}{lll}
\boldsymbol{\theta}_{1} & \ldots & \boldsymbol{\theta}_{k}
\end{array}\right]\right)
$$

which produces a solution to (11) for the selected grid parameters and outputs corresponding complex reflectivities, perturbations and the phase errors for each cross-range position. The residual error is then calculated using the phase corrected measurements and perturbed basis vectors. It is important to note that the solver $\mathbb{S}(\cdot)$ is not dependent specifically on OMP and can be integrated into any algorithm that provides a suitable estimation of the correct grids.

Even after an estimate for the $k$-grid positions are provided by the OMP, the optimization problem in (11) is still nonconvex and care is needed to obtain estimates for the complex reflectivities $\boldsymbol{\alpha}_{k}$, perturbations from the given grid centers $\delta \boldsymbol{\theta}_{k}$ and phase errors $\phi_{i}$. For this purpose, an iterative optimization approach where each of these parameters is optimized separately while keeping others fixed and this is done iteratively until a convergence criteria is met.

First; the reflectivity vector $\boldsymbol{\alpha}$ is estimated by using

$$
\boldsymbol{\alpha}_{l}=\arg \min _{\alpha}\left\|\mathbf{y}-\sum_{p=1}^{k} \alpha_{p} \mathbf{g}\left(\boldsymbol{\theta}_{p, l}\right)\right\|_{2}
$$

where $l$ is the index of the iterations of the solver $\mathbb{S}(\cdot)$ and $\boldsymbol{\theta}_{p, l}$ is the vector of $\boldsymbol{\theta}_{p}$ at iteration $l$. Using the current grid parameters and reflectivity values, the corresponding phase errors for each pulse are estimated as

$$
\hat{\phi}_{i}=\angle \boldsymbol{\alpha}_{i, l}^{H} \mathbf{G}_{i}^{H} \mathbf{y}_{i}
$$

where $i$ represents the $i$ th pulse. Phase error correction on the measurements is performed using the phase estimates in (14) as $\widehat{\mathbf{y}}_{i}=e^{-j \hat{\phi}_{i}} \mathbf{y}_{i}$, where $\hat{\mathbf{y}}_{i}$ is the phase error corrected measurement vector. Next, the grid perturbation parameters are updated and parameter perturbation is done as $\boldsymbol{\theta}_{p, l+1}=\boldsymbol{\theta}_{p, l}+\delta \boldsymbol{\theta}_{p, l}$, where $\left[\delta \boldsymbol{\theta}_{1, l} \ldots \delta \boldsymbol{\theta}_{k, l}\right]$ is obtained 
as the solution to

$$
\begin{array}{r}
\min _{\substack{\delta f_{1, p}:\left|\delta f_{1, p}\right| \leq \Delta_{1} / 2 \\
\delta f_{2, p}:\left|\delta f_{2, p}\right| \leq \Delta_{2} / 2}} \| \hat{\mathbf{y}}-\sum_{p=1}^{k} \alpha_{p, l} \mathbf{g}\left(f_{1, p, l}\right. \\
\left.\quad+\delta f_{1, p}, f_{2, p, l}+\delta f_{2, p}\right) \|_{2}
\end{array}
$$

The solution of (13) can be found by using the well known least squares techniques. Equation (15) is a constrained nonlinear optimization problem which can be solved with a gradient descent approach. For this purpose, the cost function in (15) can be linearized around $\boldsymbol{\theta}_{p, l}=$ $\left(f_{1, p, l}, f_{2, p, l}\right)$. Since, the required search is over a small sized spatial area, this linearization approach works well in practice and helps reduce the complexity of the required optimization. Specifically, $\mathbf{g}\left(f_{1, p, l}+\delta f_{1, p}, f_{2, p, l}+\delta f_{2, p}\right)$ is approximated by using the first-order Taylor series as

$$
\begin{aligned}
& \mathbf{g}\left(f_{1, p, l}+\delta f_{1, p}, f_{2, p, l}+\delta f_{2, p}\right) \approx \mathbf{g}\left(f_{1, p, l}, f_{2, p, l}\right) \\
& \quad+\frac{\partial \mathbf{g}}{\partial f_{1, p, l}} \delta f_{1, p}+\frac{\partial \mathbf{g}}{\partial f_{2, p, l}} \delta f_{2, p} .
\end{aligned}
$$

By using (16) and dropping constraints (15) can be written as

$$
\begin{aligned}
{\left[\delta \boldsymbol{\theta}_{1, l} \ldots \delta \boldsymbol{\theta}_{k, l}\right] } & =\underset{\mathbf{u}}{\arg \min } \mathbf{J}_{R}(\mathbf{u}) \\
& =\underset{\mathbf{u}}{\arg \min }\left\|\boldsymbol{r}_{l}-\mathbf{B}_{l} \mathbf{u}\right\|_{2}^{2}
\end{aligned}
$$

where $\boldsymbol{r}_{l}=\hat{\mathbf{y}}-\sum_{p=1}^{k} \alpha_{p, l} \mathbf{g}\left(\boldsymbol{\theta}_{p, l}\right)$ is the orthogonal residual, and $\mathbf{B}_{l} \in \mathcal{C}^{N \times 2 k}$ is the matrix holding the weighted partial derivatives at the point of linearization

$$
\begin{aligned}
& \mathbf{B}_{l}=\left[\Delta_{1} \alpha_{1, l} \frac{\partial \mathbf{g}}{\partial f_{1,1, l}}, \ldots, \Delta_{1} \alpha_{k, l} \frac{\partial \mathbf{g}}{\partial f_{1, k, l}},\right. \\
& \left.\Delta_{2} \alpha_{1, l} \frac{\partial \mathbf{g}}{\partial f_{2,1, l}}, \ldots, \Delta_{2} \alpha_{k, l} \frac{\partial \mathbf{g}}{\partial f_{2, k, l}}\right]
\end{aligned}
$$

and $\mathbf{u}=\left[\delta f_{1,1}, \ldots, \delta f_{1, k}, \delta f_{2,1}, \ldots, \delta f_{2, k}\right]^{T} \in \mathfrak{R}^{2 k \times 1}$ holds updates of the $l$ th iteration. Because of the use of normalization by the gridsize, entries of $\mathbf{u}$ are unitless. Along with the updates in $\boldsymbol{\theta}_{p, l}, \mathbf{B}_{l}$ is also updated at each iteration.

To improve the accuracy of the search, at each step, the parameters are updated in the direction of descent dictated by the negative of the gradient as long as the updates remain in the same grid cell.

For the cost function in (17), the negative gradient of $J$ at $\mathbf{u}=\mathbf{0}$ is $-\left.\nabla_{\mathbf{u}} J(\mathbf{u})\right|_{\mathbf{u}=\mathbf{0}}=\operatorname{Re}\left\{2 \mathbf{B}_{l}^{H} \boldsymbol{r}_{l}\right\}$. Then, the main objective stated in (15) has the alternating gradient descend solution and it is given by

$$
\begin{aligned}
\boldsymbol{\alpha}_{l} & =\left[\mathbf{g}\left(\boldsymbol{\theta}_{1, l}\right) \mathbf{g}\left(\boldsymbol{\theta}_{2, l}\right) \ldots \mathbf{g}\left(\boldsymbol{\theta}_{k, l}\right)\right]^{\dagger} \mathbf{y} \\
\boldsymbol{\theta}_{p, l+1} & =\boldsymbol{\theta}_{p, l}+\boldsymbol{\mu}_{p, l} \operatorname{Re}\left\{\mathbf{B}_{l}^{H} \boldsymbol{r}_{l}\right\}
\end{aligned}
$$

where $\boldsymbol{\mu}_{p, l}$ is the properly chosen step size parameter. At this point, updates are checked to ensure the grid constraints are satisfied. Cost function is differentiable and search domain is bounded to a single grid cell. Therefore, gradient
TABLE II

Proposed Solver $\widehat{\mathbb{S}}(\cdot)$

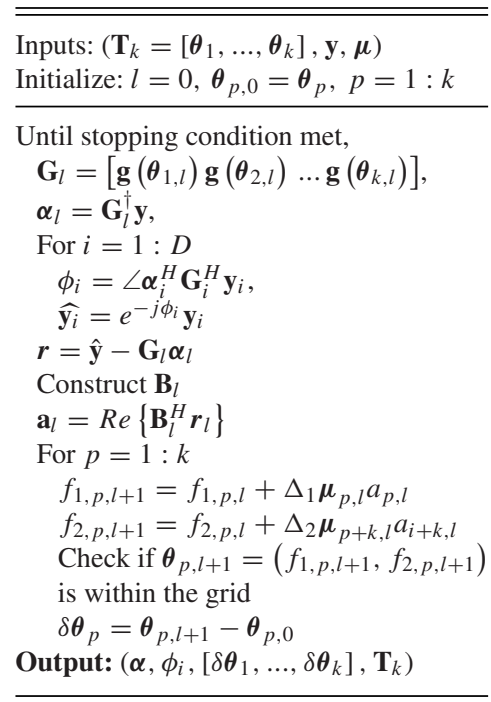

descent always converges to a local minima of the cost function in the search domain [28]. If the search domain were covering multiple grid cells, higher order approximations would be necessary. In Table II, algorithmic structure of this iterative optimization approach is given, that will be referred to as $\widehat{\mathbb{S}}(\cdot)$. In Table I, $\widehat{\mathbb{S}}(\cdot)$ is used as a part of the OMP-based method, which is capable of handling the off-grid problem and autofocus simultaneously.

Robust criteria for the termination of the iterations for $\widehat{\mathbb{S}}(\cdot)$ in Table II can be determined as a function of $\boldsymbol{r}_{l}, \boldsymbol{\theta}_{p, l}$ and $\left\|\mathbf{B}_{l}^{H} \boldsymbol{r}_{l}\right\|_{2}$ or a combination of these variables. In this study, the iterations are terminated when the norm of the residual $\left\|\boldsymbol{r}_{l}\right\|$ is less than a threshold. Although variable step sizes for $\mu$ can be chosen, the uniform grid size of the spotlight mode SAR application allows use of a constant step size for simple updates. The actual value of the step size can easily be determined, based on investigations conducted on specific SAR systems.

\section{SIMULATIONS}

In this section, the performance of the proposed PASAR algorithm is investigated and compared under various scenarios. Comparisons are performed both against classical techniques such as PFA [24], the PGA [7], and more recent sparsity-based techniques such as SDA [9], EMMP [11], and sparsity-based autofocus (SBA) [10]. Qualitative results for both simulated and real SAR system data are provided. Also quantitative comparisons on different metrics are given for various conditions.

It is important to note that the true sparse scenes contains reflectors that can be anywhere on a continuous parameter space rather than a discretized image pixels. While compared techniques generate a reflectivity image on a discrete grid, the output of PA-SAR algorithm is autofocused reflectivity and position estimates of off-grid reflectors rather than a discretized image. Hence classical mean-squareerror (MSE) metric is not a proper way of evaluating the 


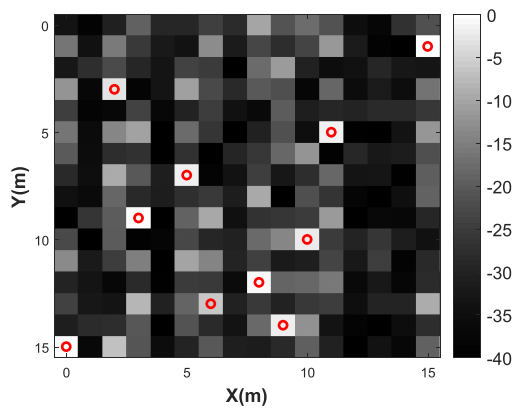

(a)

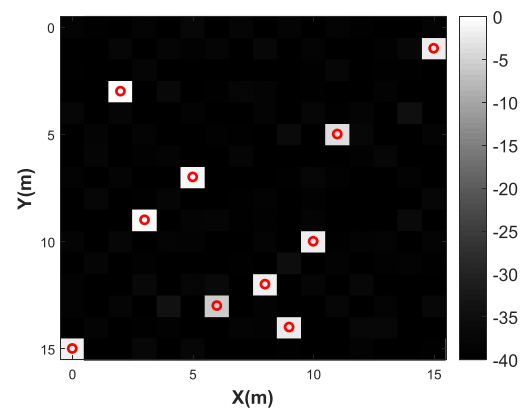

(d)

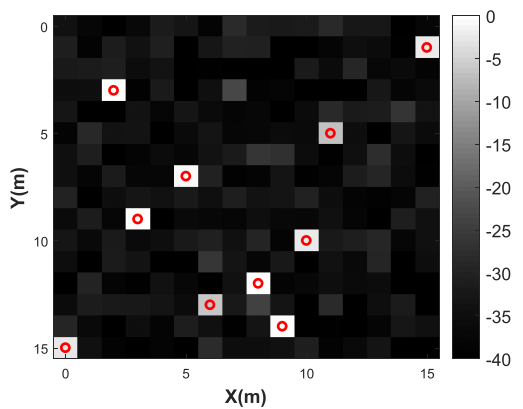

(b)

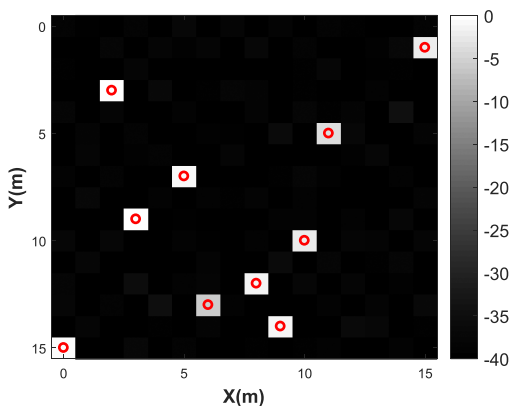

(e)

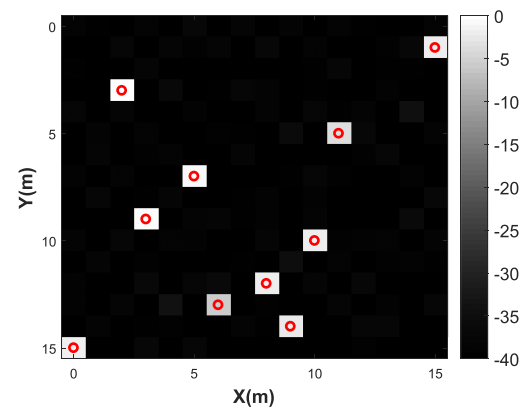

(c)

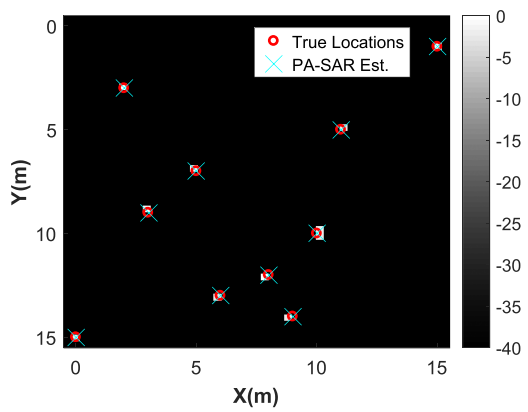

(f)

Fig. 1. Reconstructed images of SAR autofocus techniques on $(16 \mathrm{~m}) \times(16 \mathrm{~m})$ synthetic image with on-grid reflectors. The circles indicate the true locations of $K=10$ reflectors while crosses indicate PA-SAR results. Images are shown in dB scale normalized to its own maximum. (a) PFA, (b) PGA, (c) SBA, (d) SDA, (e) EMMP and (f) PA-SAR.

performance of any algorithm under off-grid target scenarios. Instead, Earth mover distance (EMD)[29], [30] metric, which is also used to measure sparse off-grid reconstructions in various applications [31], is proposed to be used in quantitative analysis of SAR image reconstructions. In addition to EMD, suitable metrics that have been used in SAR imaging such as target-to-Background Ratio (TBR) [9] and Entropy $\left.\left(\mathrm{H}_{\alpha}\right)\right)$ [32] are also calculated. The used metrics are defined as follows:

1) Earth movers distance (EMD): $P=\left(p_{i}, u_{i}\right)_{i=1}^{m}$ and $Q=\left(q_{j}, v_{j}\right)_{j=1}^{n}$ are the reconstructed and the original scenes, respectively. $p_{i}$ and $q_{j}$ denote amplitudes, $u_{i}$ and $v_{j}$ denote locations of the $i$ th and $j$ th reflectors, respectively. The EMD value is the minimum work (dust to be moved as the name implies) needed for the reconstructed scene to match the original scene. The EMD is defined as [29], [30]

$$
\operatorname{EMD}(P, Q)=\min _{F=\left\{\hat{f}_{i, j}\right\}} \frac{\sum_{i, j} \hat{f}_{i, j} d_{i, j}}{\sum_{i, j} \hat{f}_{i, j}}
$$

with constraints $\sum_{j} \hat{f}_{i, j} \leq p_{i}, \sum_{i} \hat{f}_{i, j} \leq q_{i}, \sum_{i, j} \hat{f}_{i, j}=$ $\min \left(\sum_{i} p_{i}, \sum_{j} q_{j}\right)$ and $\hat{f}_{i, j} \geq 0 . d_{i, j}$ is the ground $\mathrm{Eu}-$ clidean distance between reflectors $i$ and $j . F=\left\{\hat{f}_{i, j}\right\}$ denotes set of flows. The flow $\hat{f}_{i, j}$ denotes the amount transported from the $i$ th reflector to $j$ th reflector. EMD is calculated as defined in [30].
2) Entropy: The entropy $\left(\mathrm{H}_{\alpha}\right)$ [32] is related to the sharpness of the image and defined as

$$
H_{\alpha}=-\sum_{i} p_{i} \log _{2} p_{i}
$$

where $p$ contains the histogram counts of the scene $\boldsymbol{\alpha}$. For sharper images, entropy value is smaller.

3) TBR is defined in [9] as

$$
\mathrm{TBR}=20 \log _{10}\left(\frac{\max _{i \in T}\left|\hat{\boldsymbol{\alpha}}_{i}\right|}{\frac{1}{I_{B}} \sum_{j \in B}\left|\hat{\boldsymbol{\alpha}}_{j}\right|}\right)
$$

where $T$ and $B$ denote the pixels for the target and the background, respectively. $I_{B}$ is the number of the background pixels.

For the compared techniques, it is preferable to result in lower values of EMD and $H_{\alpha}$ and higher values of TBR for better reconstructions. The observed results are presented in the following three sections being, qualitative simulations, quantitative comparisons, and real data results.

\section{A. Qualitative Simulations}

To demonstrate and compare the improvements in visual image quality, qualitative results for the proposed PASAR technique and the compared techniques have been presented on various synthetic scenes. Synthetic SAR data for a $(16 \mathrm{~m}) \times(16 \mathrm{~m})$ scene, with $K=10$ reflectors is generated with the SAR simulation parameters selected as; carrier frequency $f_{c}=10 \mathrm{GHz}$, FM chirp rate $6 \cdot 10^{12} \mathrm{~Hz}^{2}$, pulse duration $T_{p}=2.5 \times 10^{-5} \mathrm{~s}$. Target reflectivities are 


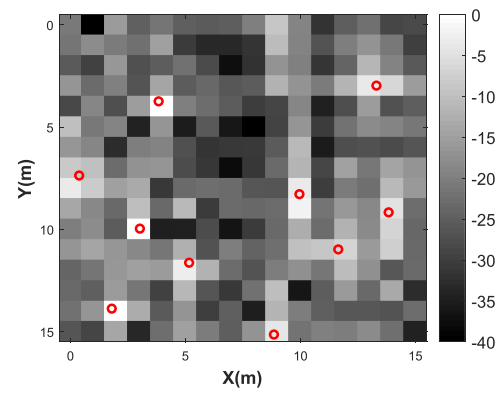

(a)

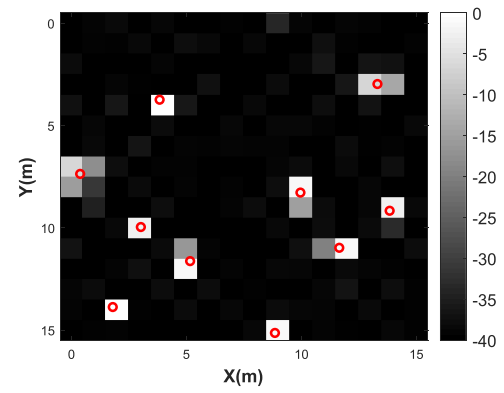

(d)

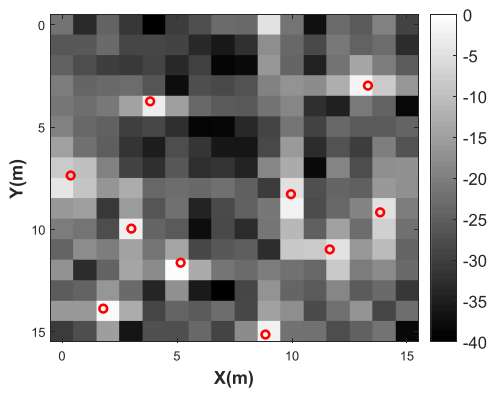

(b)

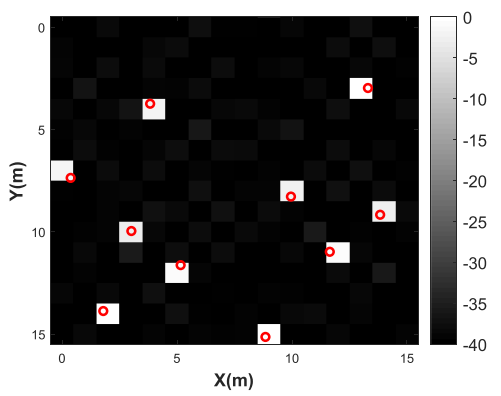

(e)

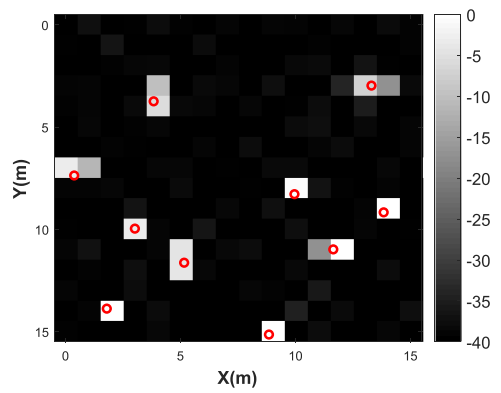

(c)

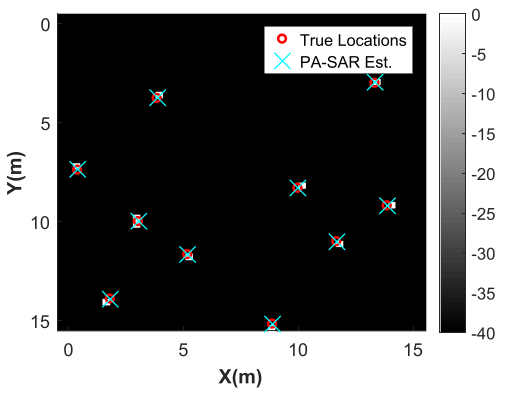

(f)

Fig. 2. Reconstructed images of SAR autofocus techniques on $(16 \mathrm{~m}) \times(16 \mathrm{~m})$ synthetic image with off-grid reflectors. The circles indicate the true locations of $K=10$ reflectors while crosses indicate PA-SAR results. Images are shown in dB scale normalized to its own maximum. (a) PFA, (b) PGA, (c) SBA, (d) SDA, (e) EMMP, and (f) PA-SAR.

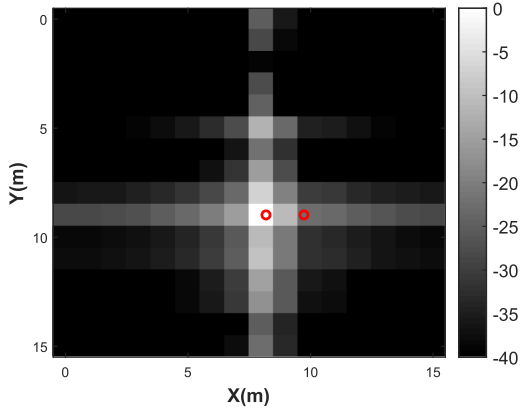

(a)

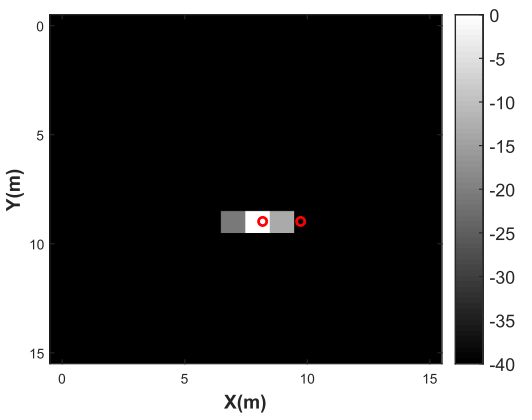

(d)

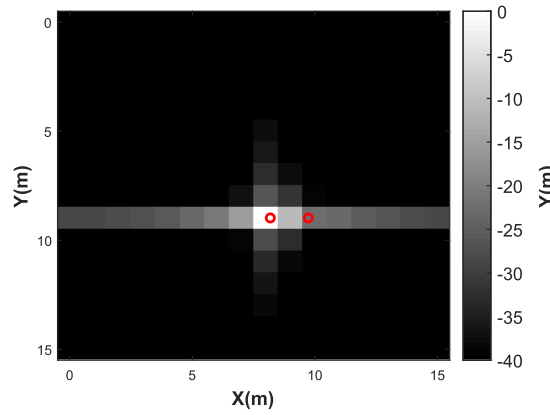

(b)

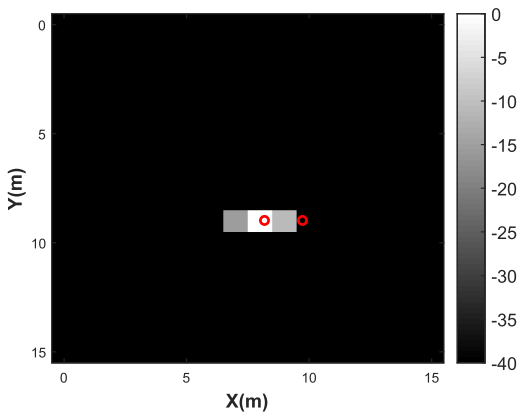

(e)

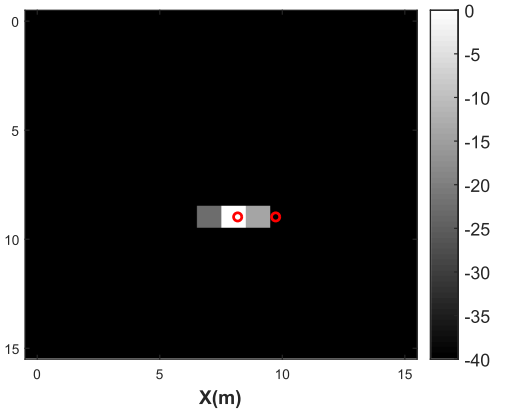

(c)

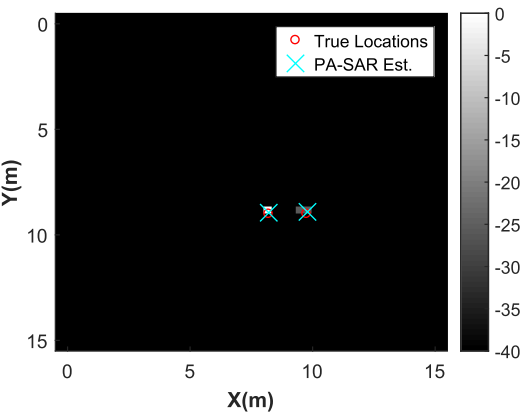

(f)

Fig. 3. Reconstructed images of SAR autofocus techniques on the synthetic scene with two closely placed off grid point reflectors. The circles indicate the true locations of the reflectors while crosses indicate PA-SAR results. Images are shown in dB scale normalized to its own maximum. (a) PFA, (b) PGA, (c) SBA, (d) SDA, (e) EMMP (f) PA-SAR.

generated randomly where amplitudes are drawn from $N(10,1)$ and phases are uniform in $[0,2 \pi]$. Both azimuth and the range resolutions are set to be $1 \mathrm{~m}$. 1-D phase error is injected to the data due to uncompansated platform motion and complex WGN is added to the data as observation noise such that SNR is $10 \mathrm{~dB}$.
For the first scenario, all targets are placed exactly on the grid. The obtained results for all the tested techniques compared to the uncompansated case of PFA are shown in Fig. 1. True target locations are shown with circles while crosses indicate PA-SAR results. Since PA-SAR generates target position and reflectivity estimates, to have an image 


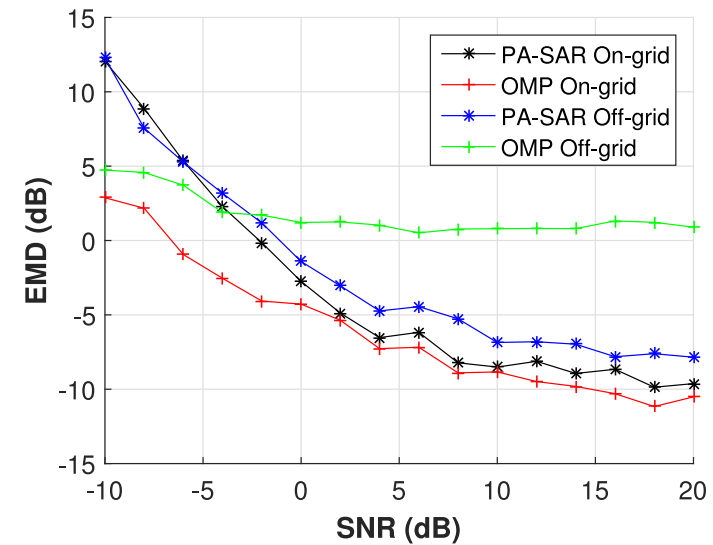

(a)

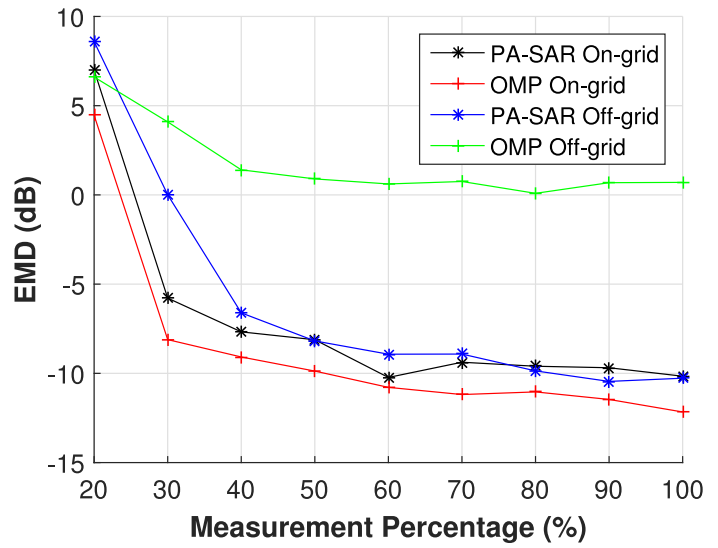

(b)

Fig. 4. Comparisons with OMP for both on-grid and off-grid scenes (a) EMD for varying SNR levels when 50\% of the measurements are used, (b) EMD at $\mathrm{SNR}=20 \mathrm{~dB}$ for varying levels of measurement percentages.

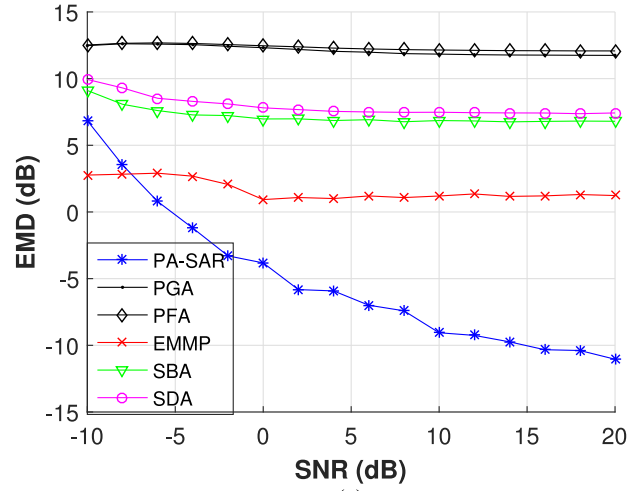

(a)

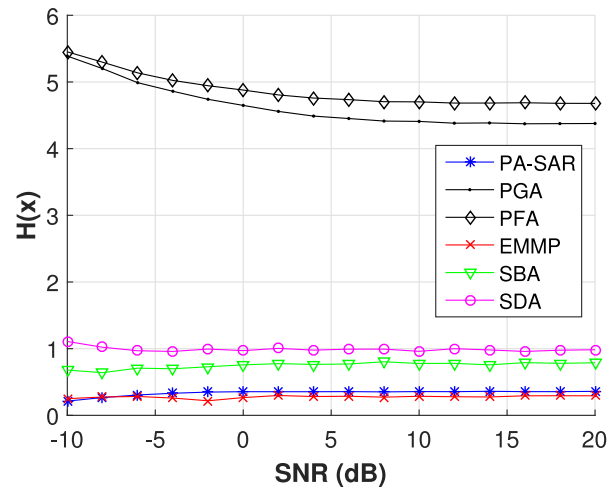

(c)

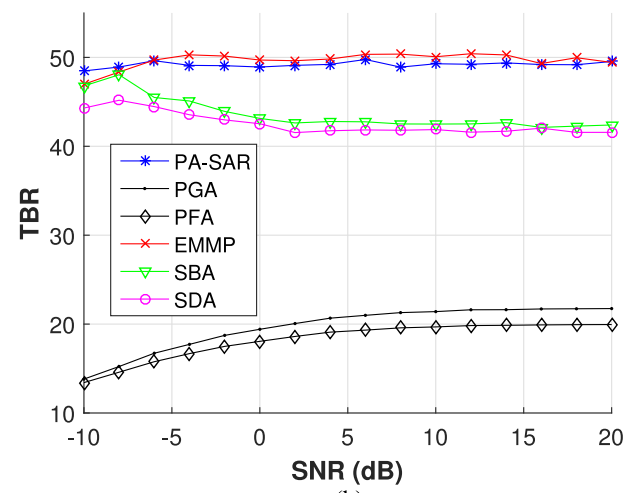

(b)

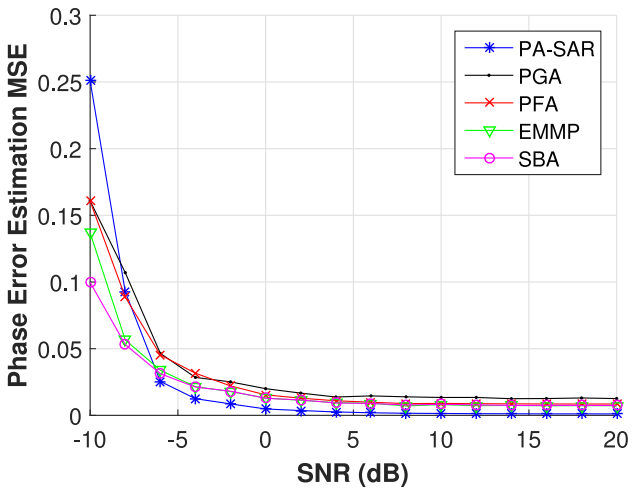

(d)

Fig. 5. (a) EMD, (b) TBR, (c) $\mathrm{H}_{\alpha}$, and (d) MSE on phase error estimation for different SNR levels.

illustration, targets positions are mapped to the nearest pixels with respective target reflectivity values. For this case where all the targets are exactly on the grid, it is seen that all SBA techniques reconstruct the sparse scene with similar performance, which looks much better than classical PGA.

For a similar setting as in the first scenario if the reflectors are only placed randomly off-grid, the performance of the compared techniques now become as shown in Fig. 2. Although still sparsity-based techniques perform much better results compared to PGA, for the techniques that does not consider off-grid targets, the images are disturbed compared to the on-grid results shown in Fig. 1. On the other hand, proposed PA-SAR algorithm can autofocus and image the sparse scene under off-grid case as well.

It can be seen from Fig. 2 that off-grid targets mainly effect the neighboring pixels due to sinc-like response of each scatterer. This is observed in SAR imaging when high reflectivity man-made objects create high sidelobes extending several pixels and possibly shadowing smaller reflectivity targets in their vicinity. To demonstrate the effectiveness of PA-SAR for closely placed reflectors that have a relatively high ratio of target reflectivities another simulation is performed. Two targets with absolute target reflectivity ratio of 20 to 1 are both placed off the grid and close to each other. The obtained results for compared techniques are shown in 


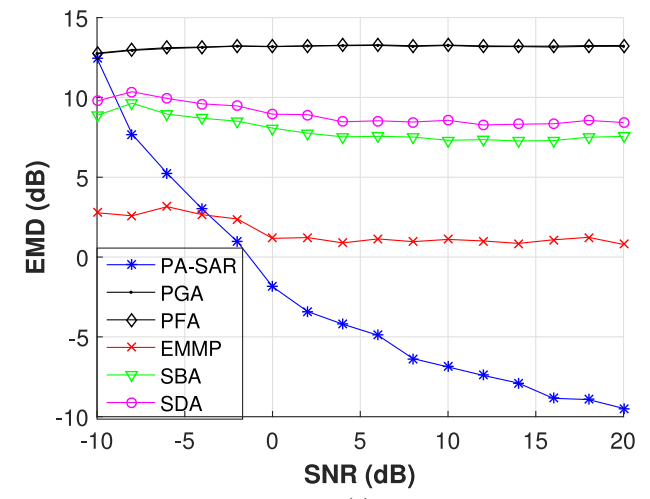

(a)

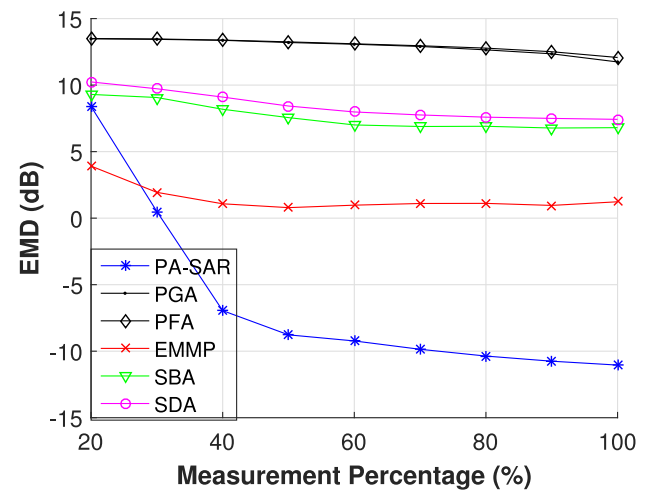

(b)

Fig. 6. (a) EMD for varying SNR levels when $50 \%$ of the measurements are used, and (b) EMD at SNR $=20$ dB for varying levels of measurement percentages.

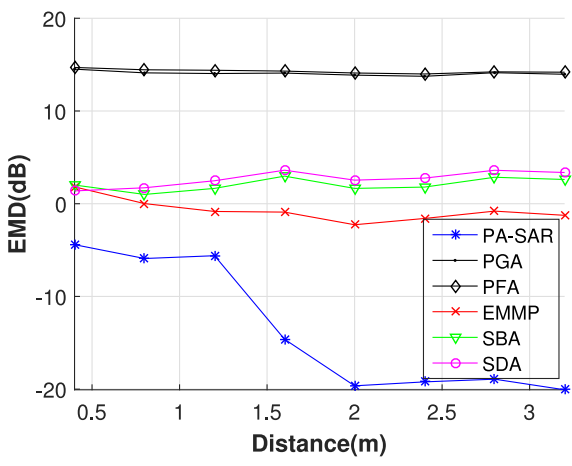

Fig. 7. Resolution performance analysis.

Fig. 3. It can be seen that while other sparsity-based techniques mainly image the dominant scatterer and its sidelobes and miss the weak target, PA-SAR can image the two targets resolving them separately, since it jointly solves for both phase and off-grid errors. This advantage is one of the major novelties of the proposed PA-SAR technique.

\section{B. Quantitative Analysis}

In this part, the proposed PA-SAR technique is compared with the existing autofocus techniques in terms of EMD, TBR, and Entropy metrics for varying levels of SNR and measurement levels. To achieve a quantitative comparison 50 independent realizations of $(16 \mathrm{~m}) \times(16 \mathrm{~m})$ synthetic scenes with randomly located $K=10$ off grid reflectors are generated. For each realization, a different 1-D cross-range-varying phase error is added to the synthetic data. First different SNR levels in the range of -10 to 20 $\mathrm{dB}$ are tested using the full SAR data. For each tested SNR level, independent realizations of complex WGN noise is added to each scene realization and the tested metrics are computed and average results are given.

To demonstrate the performance of the perturbation procedure, results of the PA-SAR and the OMP with phase error correction are compared on both on-grid and off-grid scenarios. OMP reconstructions are generated with a range of prior parameters and the one with the minimum EMD result is selected. Therefore, OMP has the minimum EMD results with any prior parameter selection. Results are given in the Fig. 4. For scenes with on-grid targets OMP yields slightly better results due to its choice of vectors that perfectly match the target grid locations. For off-grid case, PASAR performance remains unperturbed, meanwhile OMP results degrade considerably compared to the on-grid case. This demonstrates the effectiveness of the proposed PASAR algorithm.

The results of the tested metrics as a function of SNR are shown in Fig. 5. Comparisons in terms of EMD metric is shown in Fig. 5(a), where proposed PA-SAR algorithm has superior performance for SNR levels higher than -5 $\mathrm{dB}$. This is because EMD metric takes into account both the target reflectivities and off-grid target positions, and only PA-SAR algorithm does joint phase and off-grid error minimization. Also, sparsity-based techniques show lower EMD results compared to PFA and PGA algorithms for all SNR values. It is important to note that compared techniques of SDA, SBA, and EMMP require different prior parameters to work with and their performances change with the selection of these parameters. In the results shown in Fig. 5, the compared techniques has been tested with a range of their prior parameters and the one that gives the minimum EMD result is selected. Hence the EMD results for the compared techniques are the lowest values these techniques can achieve with any prior parameter selection. For the TBR and entropy results shown in Fig. 5(b) and (c), respectively, all sparsity-based techniques perform significantly better than PFA and PGA techniques, where within the sparsity-based techniques, PA-SAR and EMMP show slightly better performances. The MSE on the phase error estimation all autofocus techniques behave similarly where PA-SAR algorithm has slightly lower MSE values for SNR levels higher than $-5 \mathrm{~dB}$.

To observe the performances of the compared techniques under lower number of measurements another simulation is performed. The EMD results for varying levels of SNR when only $50 \%$ of the measurements are randomly taken is shown in Fig. 6(a). It can be seen that proposed PA-SAR algorithm still achieves lower EMD values under reduced number of measurements. Fig. 6(b) shows EMD results at a fixed SNR of $20 \mathrm{~dB}$ and a fixed sparsity level of $K=10$ for varying measurement levels. Although de- 


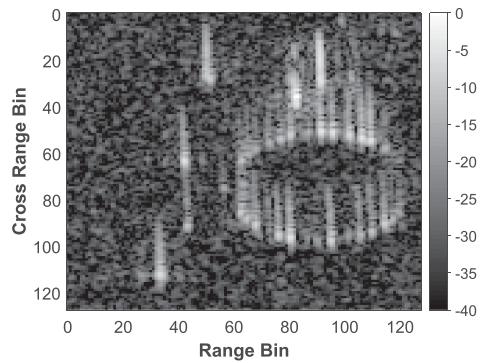

(a)

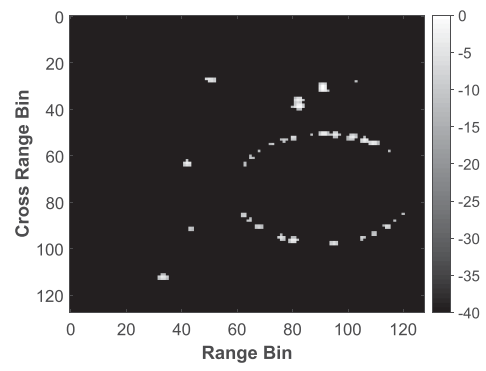

(d)

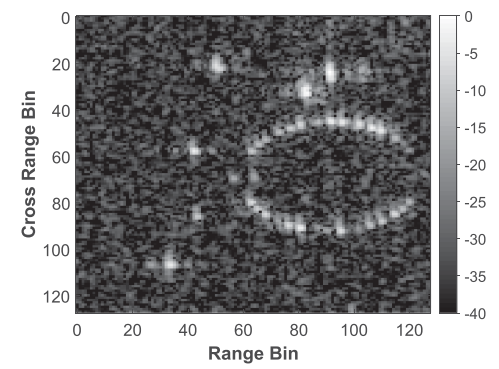

(b)

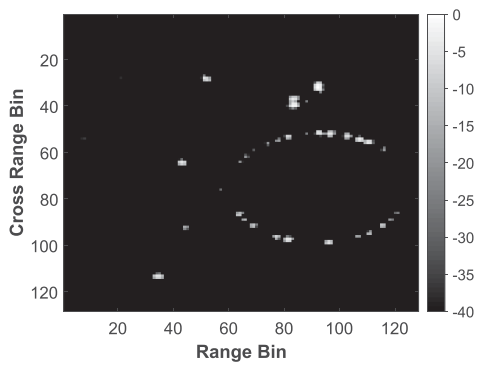

(e)

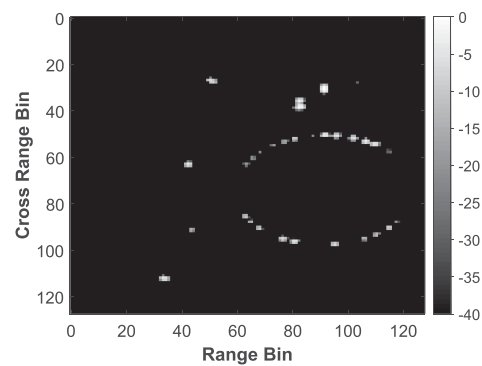

(c)

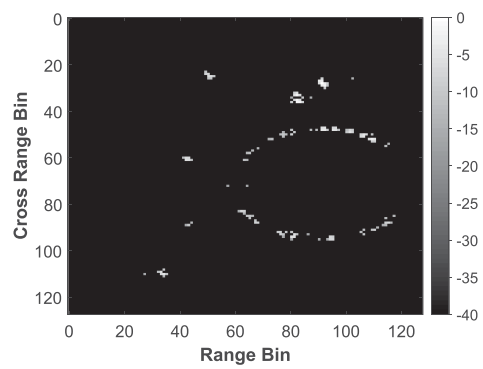

(f)

Fig. 8. Joint autofocus and SAR imaging results of compared techniques on $128 \times 128$ pixels real data obtained by SARPER-ASELSAN system with 33\% subsampled measurements. (a) PFA, (b) PGA, (b) PGA, (d) EMMP, (e) SDA, and (f) PA-SAR.

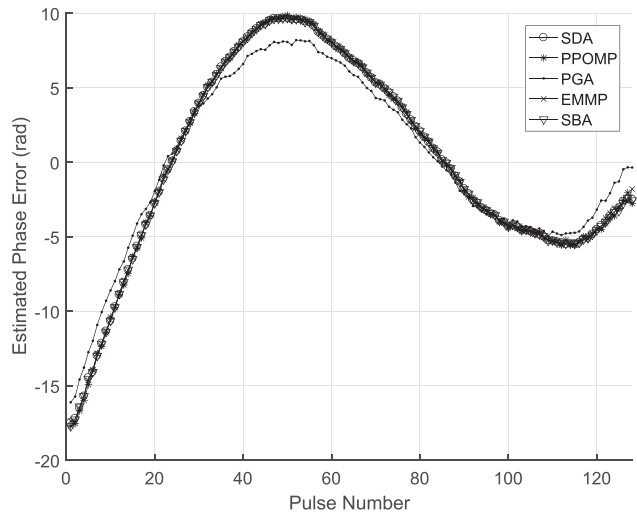

Fig. 9. Phase error estimates of real data for the compared techniques with $33 \%$ subsampled measurements.

creasing number of measurements increases EMD values, it is observed that proposed PA-SAR technique results in lower EMD values with measurement numbers more than $40 \%$ of the full SAR data. Since EMD metric is affected by off-grid estimation performance further decrease of measurement numbers increases EMD of PA-SAR bringing its performance similar to compared techniques. This shows that PA-SAR can provide both good reflectivity and off-grid location estimates with measurement levels of $40 \%$ or more at the tested SNR and sparsity levels.

Another simulation scenario is performed for the resolution performance analysis of the compared techniques. In this set of simulations, $16 \mathrm{~m} \times 16 \mathrm{~m}$ synthetic off-grid target scenes are generated for two equal strength reflectors with random locations. The performances of the compared techniques are observed for different ground distances between the two reflectors. For each separation between the targets, 50 independent target scenes are generated and their corre- sponding reconstructions are obtained. For each realization, a different 1-D cross-range-varying phase error is added to the synthetic data. To observe the effect of the target separation on the performance of the compared techniques, reconstructions are obtained at a relatively high SNR of 20 dB. In Fig. 7, averaged performance results are given as a function of separation distance. Although, performance of PA-SAR increases after the resolution level $(1 \mathrm{~m})$, it can be seen that PA-SAR gives better results than the compared methods at all the separation distances.

\section{Real Data Results}

The compared algorithms are also evaluated on actual SAR system complex data obtained from SARPER airborne SAR system developed by ASELSAN- [33]. Complex data are obtained over a test region where a number of experimental corner reflectors are placed. The autofocused images for the compared techniques are given in Fig. 8 and phase error estimates are shown in Fig. 9. Only 33\% of the full data are used for all sparsity-based techniques and the subsampling is performed pseudo-randomly as described in [14]. All sparsity-based techniques are terminated with the same criteria.

It can be seen that all sparsity-based techniques provide autofocused and sparse images compared to PFA and PGA. Since targets are not only point targets, the obtained images are mostly similar for compared sparsity-based techniques; but PA-SAR provides better separation between close targets where compared techniques seem to overlap these. For the phase error estimate, all sparsity-based techniques agree with each other where only PGA differs.

Two more actual SAR system complex dataset are also used to demonstrate the PA-SAR performance. The complex dataset were obtained from SARPER -airborne SAR 


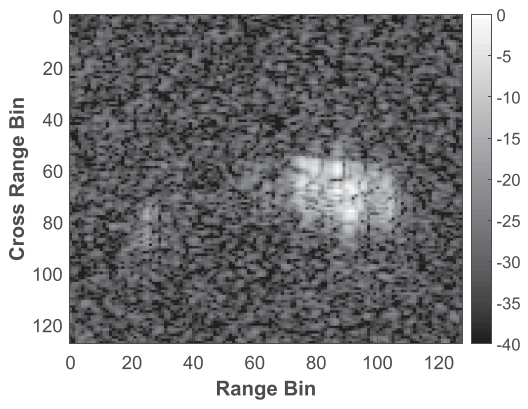

(a)

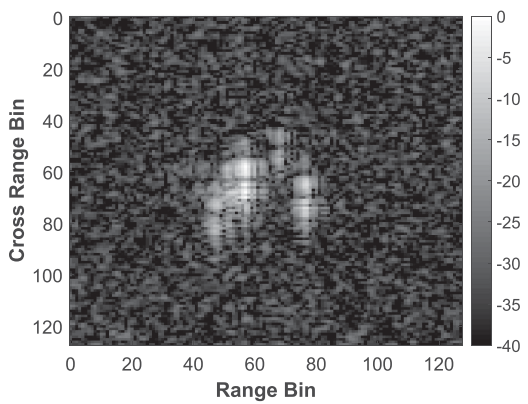

(d)

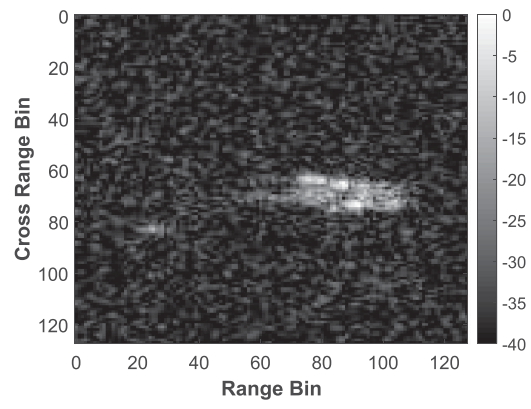

(b)

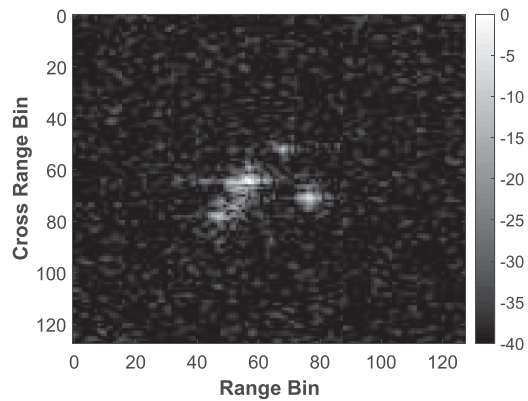

(e)

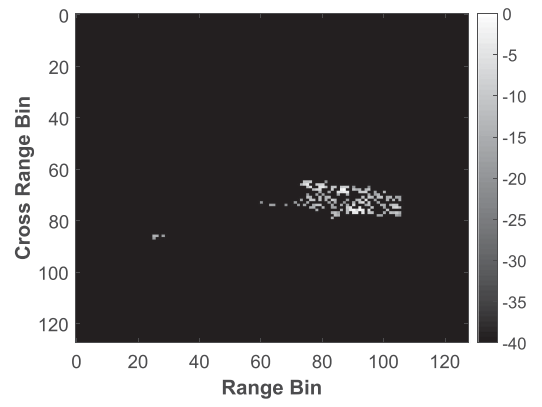

(c)

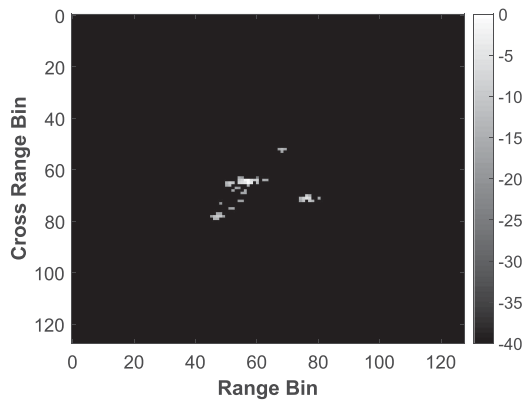

(f)

Fig. 10. Joint autofocus and SAR imaging results of PA-SAR on two different $128 \times 128$ pixels real data sets obtained by SARPER ${ }^{\text {TM }}$ - ASELSAN system with 33\% subsampled measurements. (a), (b), (c) Imaging results of man-made object 1, (d), (e), (f) Imaging results of man-made object 2. (a) PFA, (b) PGA, (c) PA-SAR, (d) PFA, (e) PGA, and (f) PA-SAR.

system developed by ASELSAN-[33]. Only 33\% of the full data are used for PA-SAR and the results are given in Fig. 10. PFA reconstructs defocused images while PGA successfully performs autofocus. It can be seen that the PA-SAR reconstructs autofocused sparse images while preserving relevant shape details.

\section{CONCLUSION}

A PA-SAR imaging technique is developed which joinly solves for both platform motion-based phase errors and off the grid target errors. The proposed technique is an orthogonal matching pursuit-based greedy technique, where target reflectivities, platform induced phase errors, and the offgrid target location perturbations are solved iteratively. For solving off-grid target perturbations, PA-SAR technique uses a gradient descent type parameter update together with phase error correction to do the joint autofocused imaging. The technique allows reduced number of measurements and results in sparse SAR images. The results obtained by using both simulated and real SAR system data show that the proposed technique provides better SAR image reconstructions in terms of compared metrics.

\section{REFERENCES}

[1] D. Munson, J. O'Brien, and W. Jenkins

A tomographic formulation of spotlight-mode synthetic aperture radar

Proc. IEEE, vol. 71, no. 8, pp. 917-925, Aug. 1983.

[2] D. Donoho

Compressed sensing

IEEE Trans. Inf. Theory, vol. 52, no. 4, pp. 1289-1306, Apr. 2006.
[3] E. Candes, J. Romberg, and T. Tao

Robust uncertanity principles: Exact signal reconstruction from highly incomplete frequency information

IEEE Trans. Inf. Theory, vol. 52, no. 2, pp. 489-509, Feb. 2006.

[4] M. Cetin, I. Stojanovic, N. Onhon, and K. Varshney

Sparsity-driven synthetic aperture radar imaging: Reconstruction, autofocusing, moving targets, and compressed sensing IEEE Signal Process. Mag., vol. 31, no. 4, pp. 27-40, Jul. 2014.

[5] J. Ender

A brief review of compressive sensing applied to radar

In Proc. 2013 14th Int. Radar Symp., 2013, vol. 1, pp. 3-16.

[6] R. Baraniuk, M. Davenport, R. DeVore, and M. Wakin

A simple proof of the restricted isometry property for random matrices

Constructive Approx., vol. 28, no. 3, pp. 253-263, 2008.

[7] D. Wahl, P. Eichel, D. Ghiglia, and C. Jakowatz

Phase gradient autofocus - A robust tool for high resolution SAR phase correction

IEEE Trans. Aerosp. Electron. Syst., vol. 30, no. 3, pp. 827-835, Jul. 1994.

[8] R. Morrison, M. Do, and J. Munson, D.C.

Mca: A multichannel approach to sar autofocus

IEEE Trans. Image Process., vol. 18, no. 4, pp. 840-853, Apr. 2009.

[9] O. Onhon and M. Cetin

A sparsity-driven approach for joint SAR imaging and phase error correction

IEEE Trans. Image Process., vol. 21, no. 4, pp. 2075-2088, Apr. 2012.

[10] S. Kelly, M. Yaghoobi, and M. Davies

Sparsity-based autofocus for undersampled sysnthetic aperture radar

IEEE Trans. Aerosp. Electron. Syst., vol. 50, no. 2, pp. 972-986, Apr. 2014.

[11] S. Ugur, O. Arikan, and A. Gurbuz

SAR image reconstruction by expectation maximization based matching pursuit

Digit. Signal Process. vol. 37, pp. 75-84, 2015. 
C. V. Jakowatz, D. E. Wahl, P. H. Eichel, D. C. Ghiglia, and P. A. Thompson

Spotlight-Mode Synthetic Aperture Radar: A Signal Processing Approach. New York, NY, USA: Springer, 1996.

[13] S. Samadi, M. Cetin, and M. Masnadi-Shirazi Sparse representation based synthetic aperture radar imaging IET Radar, Sonar Navig., vol. 5, no. 2, pp. 182-193, Feb. 2011.

[14] S. Ugur and O. Arikan

Sar image reconstruction and autofocus by compressed sensing Digit. Signal Process., vol. 22, no. 6, pp. 923-932, Dec. 2012.

[15] T. Jihua, S. Jinping, H. Xiao, and Z. Bingchen

Motion compensation for compressive sensing SAR imaging with autofocus

In Proc. 2011 6th IEEE Int. Conf. Ind. Electron. Appl., 2011, pp. $1564-1567$.

[16] A. Gungor, M. Cetin, and E. Guven

An augmented lagrangian method for autofocused compressed sar imaging

In Proc. 2015 3rd IEEE Int. Workshop Compressed Sens. Theory Appl. Radar, Sonar Remote Sensing, 2015, pp. 1-6.

[17] Y. Chi, L. Scharf, A. Pezeshki, and R. Calderbank

The sensitivity to basis mismatch of compressed sensing in spectrum analysis and beamforming

In Proc. 6th Workshop Def. Appl. Signal Process., Lihue, HI, USA, Oct. 2009.

[18] O. Teke, A. Gurbuz, and O. Arikan

A robust compressive sensing based technique for reconstruction or sparse radar scenes

Digit. Signal Process., vol. 27, pp. 23-32, 2013.

[19] H. Zhu, G. Leus, and G. Giannakis

Sparsity-cognizant total least-squares for perturbed compressive sampling

IEEE Trans. Signal Process., vol. 59, no. 5, pp. 2002-2016, May 2011.

[20] G. Tang, B. Bhaskar, P. Shah, and B. Recht

Compressed sensing off the grid

IEEE Trans. Inf. Theory, vol. 59, no. 11, pp. 7465-7490, Nov. 2013.

[21] O. Teke, A. Gurbuz, and O. Arikan

Perturbed orthogonal matching pursuit

IEEE Trans. Signal Process., vol. 61, no. 24, pp. 6220-6231, Dec. 2013.

[22] C. Ekanadham, D. Tranchina, and E. P. Simoncelli

Recovery of sparse translation-invariant signals with continuous basis pursuit

IEEE Trans. Signal Process., vol. 59, no. 10, pp. 4735-4744, Oct. 2011.
[23] S. Camlica, A. Gurbuz, and O. Arikan

SAR image reconstruction with joint off-grid target and phase error corrections

In Proc. 2015 IEEE Int. Geosci. Remote Sens. Symp., 2015, pp. $4502-4505$.

[24] W. G. Carrara, R. Goodman, and R. Majewski Spotlight Synthetic Aperture Radar, Signal Processing Algorithms. Norwood, MA, USA: Artech House, 1995.

[25] S. Ugur

Novel Methods for SAR Imaging Problems

Ph.D. dissertation, Dept. Elect. Electron. Eng., Bilkent Univ., 2013.

[26] M. Mishali, Y. Eldar, and A. Elron

Xampling: Signal acquisition and processing in union of subspaces

IEEE Trans. Signal Process., vol. 59, no. 10, pp. 4719-4734, Oct 2011.

[27] J. Tropp and A. Gilbert

Signal recovery from random measurements via orthogonal matching pursuit

IEEE Trans. Inf. Theory, vol. 53, no. 12, pp. 4655-4666, Dec. 2007.

[28] S. Boyd and L. Vandenberghe

Convex Optimization. Cambridge, U.K.: Cambrige Univ. Press, 2004.

[29] H. Ling and K. Okada

An efficient earth mover's distance algorithm for robust histogram comparison

IEEE Trans. Pattern Anal. Mach. Intell., vol. 29, no. 5, pp. 840-853, May 2007.

[30] Y. Rubner and C. T. ans, L. J. Guibas

The earth movers distance as a metric for image retrieval Int. J. Comput. Vis., vol. 40, no. 2, pp. 99-121, 2000.

[31] L. Schmidt, C. Hegde, and P. Indyk

The constrained earth mover distance model, with applications to compressive sensing

In Proc. 10th Intl. Conf. Sampling Theory Appl., 2013, pp. $428-431$.

[32] S. Samadi, M. Cetin, and M. Masnadi-Shirazi Sparse representation based on synthetic aperture radar imaging IET Radar Sonar Navig., vol. 5, no. 2, pp. 182-193, 2011.

\section{[33] SARPER}

Aselsan SAR airborne system.

2012. [Online]. Available: http://www.aselsan.com.tr/en-us/ capabilities/radar-systems/reconnaissance-surveillance-

radars/sar-synthetic-aperture-radar.

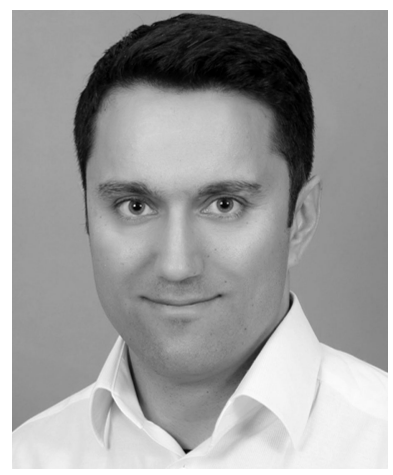

Sedat Camlica was born in Izmir, Turkey, in 1982. He received the B.Sc. degree in telecommunications engineering from Istanbul Technical University, Istanbul, Turkey, in 2006, and the M.Sc. degree in electrical and electronics engineering from the Middle East Technical University, Ankara, Turkey, in 2009. He is currently working toward the Ph.D. degree in electrical and electronics engineering at TOBB University of Economics and Technology, Ankara, Turkey.

Since 2006, he has been serving at ASELSAN A.S., Radar \& Electronic Warfare Systems Business Sector, Ankara, Turkey. His research interests include compressive sensing applications, array signal processing, target tracking, remote sensing, and imaging. 


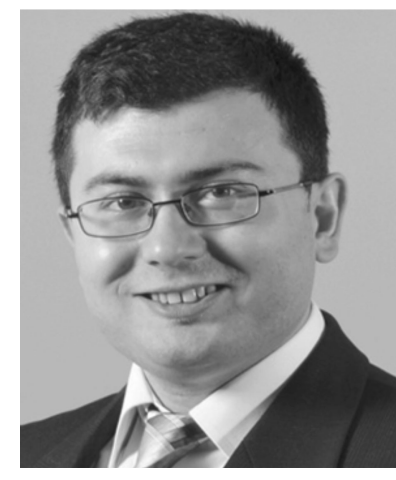

Ali Cafer Gurbuz (M'08) received the B.S. degree in electrical and electronics engineering from Bilkent University, Ankara, Turkey, in 2003, and the M.S. and Ph.D. degrees in electrical and computer engineering from Georgia Institute of Technology, Atlanta, GA, USA, in 2005 and 2008, respectively.

From 2003 to 2008, he researched multimodal landmine detection systems as a Graduate Research Assistant and from 2008 to 2009, as a Postdoctoral Fellow, all with Georgia Tech. As an Assistant Professor during 2009-2013) and an Associate Professor during 2013-2016 of electrical and electronics engineering at TOBB University of Economics and Technology, Ankara, Turkey, he worked toward an active research program on the development of sparse signal representations, compressive sensing theory and applications, radar and sensor array signal processing, machine learning and embedded signal processing. He currently in the Department of Electrical and Computer Engineering at Utah State University, Logan, UT, USA, where he is focused on developing communications, radar and sensor array system applications of multifunctional reconfigurable antennas.

He received the Best Paper Award for Signal Processing Journal in 2013 and Turkish Academy of Sciences Best Young Scholar Award in Electrical Engineering in 2014.

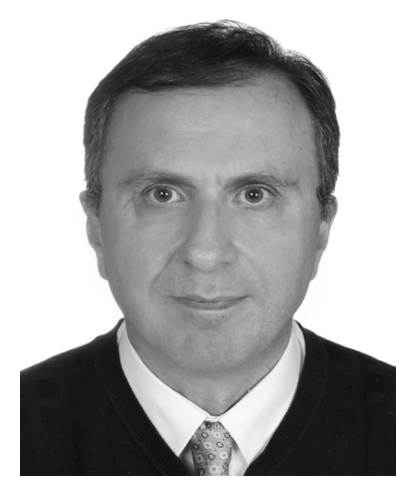

Orhan Arikan (M'91) was born in Manisa, Turkey, in 1964. He received the B.Sc. degree in electrical and electronics engineering from the Middle East Technical University, Ankara, Turkey, In 1986, and the M.S. and Ph.D. degrees in electrical and computer engineering from the University of Illinois, Urbana-Champaign, IL, USA, in 1988 and 1990, respectively.

Following his graduate studies, he was employed as a Research Scientist at Schlumberger-Doll Research Center, Ridgefield, CT, USA. In 1993, he joined the Department of Electrical and Electronics Engineering at Bilkent University, Ankara, Turkey. Since 2006, he has been a Full Professor at Bilkent University. where since 2011, he has been serving as the Chairman of the Department of Electrical and Electronics Engineering. His current research interests include statistical signal processing, time-frequency analysis, and remote sensing.

Dr. Arikan has served as the Chairman of Turkey chapter of IEEE. 